\title{
Study on Learning in Intelligent Control using ABC Type Neural Network
}

\author{
Shenglin $\mathrm{Mu}^{\mathrm{a}}$, Satoshi Shibata ${ }^{\mathrm{a}}$, Yamamoto Tomonoria \\ Kanya Tanaka ${ }^{\mathrm{b}}$, and Shota Nakashima ${ }^{\mathrm{c}}$ \\ a Graduate School of Science and Engineering, Ehime University, 3 Bunkyou-chou, Matsuyama, Ehime, Japan \\ b Department of Electronics and Bioinformatics, School of Science and Technology, \\ Meiji University, Kawasaki, Kanagawa, Japan \\ c Graduate School of Sciences and Technology for Innovation, Yamaguchi University, \\ Ube, Yamaguchi, Japan
}

*Corresponding Author: mu.shenglin.du@ehime-u.ac.jp

\begin{abstract}
In this research, a hybrid intelligent method based on NN combined with Artificial Bee Colony (ABC) to construct an auto-tuning of variable gain PID control for the control of Ultrasonic Motor (USM). The proposed method is with simple structure and capable to improve the control performance of traditional PID control. In this paper, the control performance and effectiveness of the proposed method was studied according to simulation results USM.
\end{abstract}

Keywords: Ultrasonic Motor, PID Control, Neural Network, Artificial Bee Colony.

\section{Introduction}

PID control is recognized as the most famous method in control engineering. It plays such a significant role that the method is applied in almost every industrial field. Since traditional fixed gain PID control cannot meet the requirements of auto tuning according to system characteristics, variable gain PID control is developed as an important improvement in control of automatic systems ${ }^{(1,2)}$. In our proposed method, a Neural Network (NN) is introduced as an intelligent tuning unit for PID control. Comparing with Genetic Algorithm (GA) and Fuzzy, NN performs quick and effective in control applications. However, in conventional NN with back propagation (BP) method using in control applications, the estimation of Jacobian is hard to be estimated ${ }^{(3)}$. To avoid the estimation problem, Artificial Bee Colony (ABC) is employed in the proposed method for constructing a hybrid intelligent PID control method. $\mathrm{ABC}$ is an optimization method originated from the model of bees. Since the model of ABC is simple and the estimation in it is easy, the algorithm is very attractive. In the proposed hybrid method, ABC is introduced to the weight updating in $\mathrm{NN}$. The updating procedure requires only error signal of the system, without any modeling information of the system. The mechanism of the hybrid method is simple and easy to use.

In this paper, we introduce an intelligent control method combined with $\mathrm{ABC}$ for variable gain PID control. The paper is organized as follows. PID control for USM is introduced in Sect. 2. In Sect. 3, the proposed intelligent method of hybrid NN with $\mathrm{ABC}$ is introduced. Section 4 studies the simulation results. The paper is concluded in Sect. 5 .

\section{PID Control for Ultrasonic Motor}

\subsection{Ultrasonic motor}

Different from traditional electromagnetic motors, USM is driven by friction force. The driving theory of USM is shown in Fig. 1. The concept of friction driven motor was proposed quite early. However, the motor was not realized in the early age since it was proposed. It was commercialized in 1980's by Mr. Sashida. USM was found with very outstanding features since it was commercialized. Firstly, USM offers higher torque comparing with electromagnetic motor in the same size. Secondly, USM can give high torque in low speed range without any gear, and in no power 


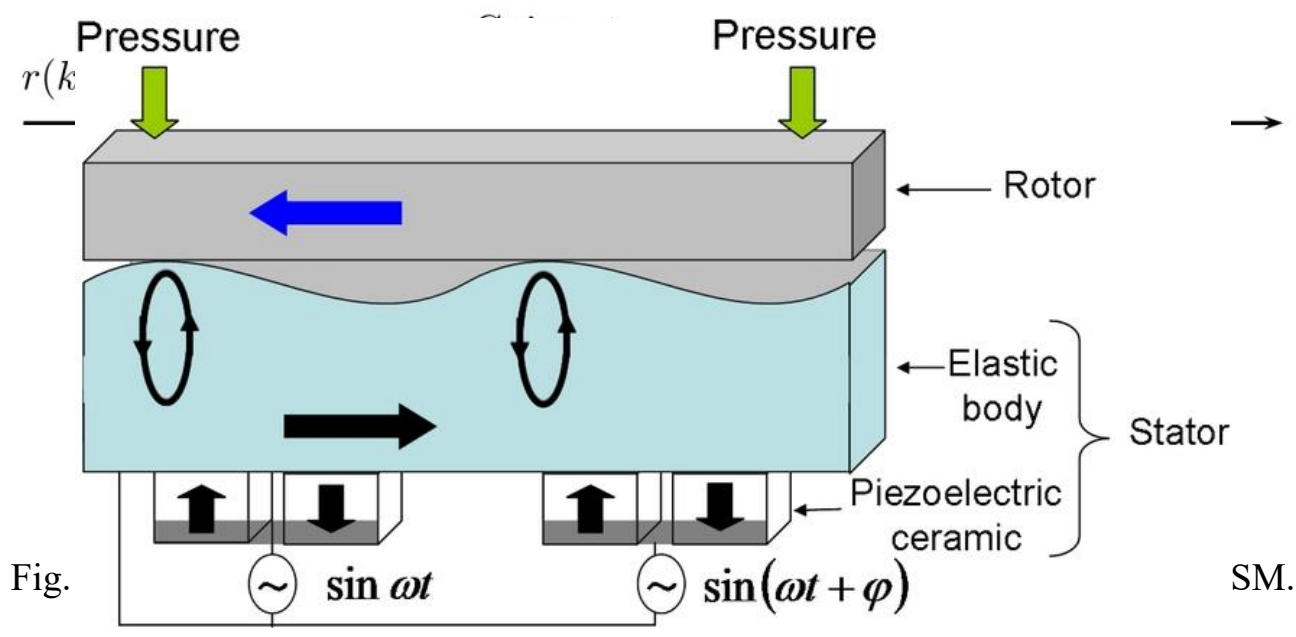

Fig. 1. Driving theory of USM.

condition, can give high retention torque. Thirdly, since USM is driven by friction generated from ultrasonic vibration, it runs very quiet offering high accuracy. Meanwhile, there is an important feature that owing to the special friction driven principle comparing with other electromagnetic motors, USM generates no electromagnetic noise and has no interferences to other electronic devices. Therefore, USM is capable to be applied in watches, autofocus cameras, cell phones, and so on. USM is still expected to be employed in more and more applications. Especially, owing to its Electromagnetic Compatibility (EMC), it is expected to be introduced more in medical and welfare fields ${ }^{(4-7)}$.

However, there are still many difficulties in the applications of USM. Firstly, the life duration of USM is not long owing to abrasion caused by the friction driving. Secondly, special driving system which is more complex than that of traditional electromagnetic motors has to be applied. For control of USM, the characteristics of USM will change according to condition changes of temperature, humidity and load and so on. Meanwhile, since USM is driven by friction force, there is non-linearity in the control of it. We do not have exact mathematic model of USM.

\subsection{PID control for ultrasonic motor}

Basic scheme of PID control for USM can be illustrated as Fig. 2 shows. In the scheme, $r(k), u(k)$, and $y(k)$ are the object input, the input and the output, respectively. $G_{P I D}\left(z^{-1}\right)$ is the PID controller. The input $u(k)$ is synthesized as

$$
\begin{gathered}
u(k)=u(k-1)+\left(K_{P}+K_{I}+K_{D}\right) e(k)-\left(K_{P}+\right. \\
\left.2 K_{D}\right) e(k-1)+K_{D} e(k-2)
\end{gathered}
$$

where $K_{P}, K_{I}$ and $K_{D}$ are the gains of the PID controller. The error denoted by $e(k)$ expressed as

$$
e(k)=r(k)-y(k)
$$

The PID controller $G_{P I D}\left(z^{-1}\right)$ can be denoted as the following equation shows.

$$
G_{P I D}\left(z^{-1}\right)=\frac{K_{P}\left(1-z^{-1}\right)+K_{I}+K_{D}\left(1-z^{-1}\right)^{2}}{1-z^{-1}}
$$

In this research, the prosed intelligent method was proposed as Fig. 3 shows. The scheme is designed for tuning PID gains automatically to achieve good performance in USM control. In previous research, PID control which was proved to work well even without any mathematic models employed.

\section{Intelligent PID control for ultrasonic motor}

In this paper, neural network has been introduced as the main intelligent unit based on PID control of ultrasonic motor. The proposed scheme is a fixed structure as shown in

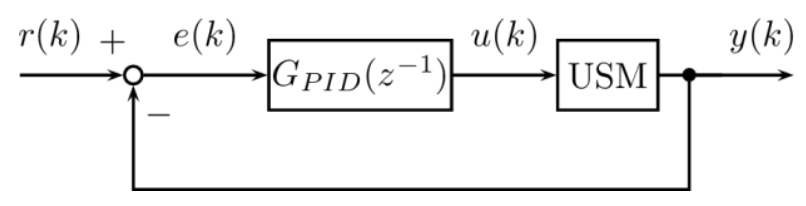

Fig. 2. Block diagram of PID control for USM. 


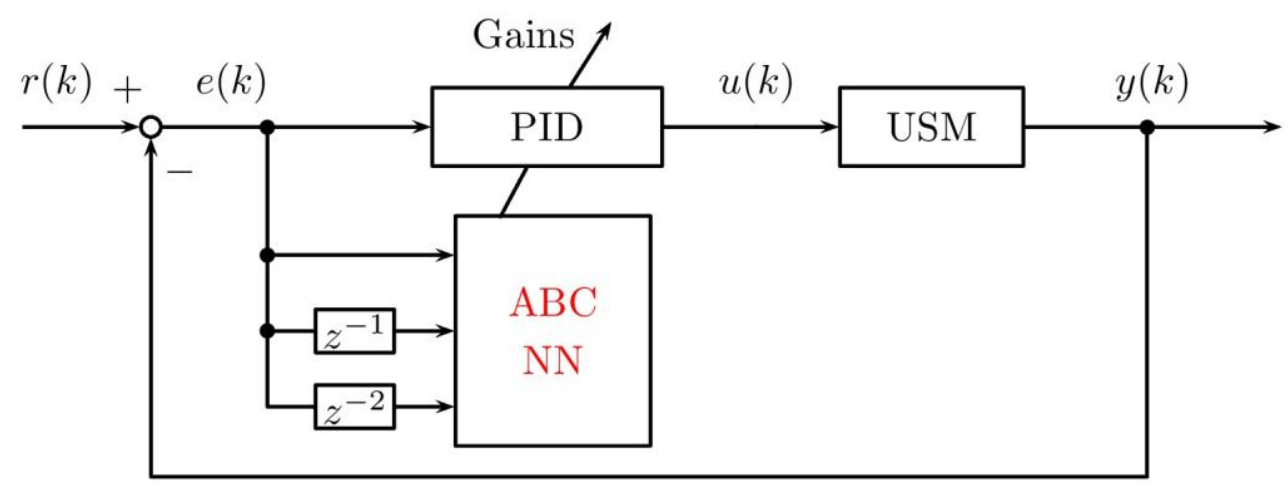

Fig. 3. Proposed scheme of ABC type NN method based on PID control for USM.

Fig. 4. The input of $\mathrm{NN}$ is the discrete time error signal. There are 6 neurons in the hidden layer. The output layer contains 3 neurons estimating variation of the PID gains. In the scheme, the weights between input layer and hidden layer, and the ones between hidden layer and output layer are updated by $\mathrm{ABC}$. The learning is designed to be implemented by $\mathrm{ABC}$ algorithm.

ABC algorithm was developed by Karaboga ${ }^{(8)}$. It simulates the food foraging behavior of honey bees. In ABC, the food source for bees are considered as solutions. Three types of bees are modeled in $\mathrm{ABC}$ algorithm, the employed, the onlooker and the scout bees. ABC colony consists equal number of employed and onlooker bees. Employed bees explore search space for food according to the information in their memories. Onlooker bees obtain the information from employed bees in the hive to select food sources for further extraction of nectar. If thenectar quantity in food source is low or exhausted, then scout bee randomly finds a new food source in search space.

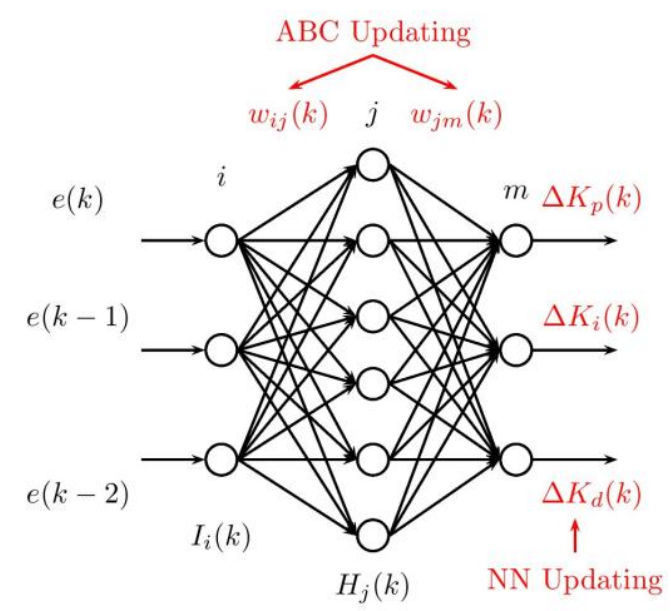

Fig. 4. Topological structure of ABC type NN.
In this research, the possible solutions of $\mathrm{NN}$ weight of are initialized with random number firstly. To all the solutions $\boldsymbol{x}_{\boldsymbol{i}}(i=1,2, \ldots, N)$, new possible solutions $\boldsymbol{v}_{\boldsymbol{i}}(i=1,2, \ldots, N)$ are generated using the random selection shown in Eq. (4). They are the real number vector with the same dimension. $i$ is the number to identify the individual, $j$ is the number of dimensions. According to greedy selection shown in Eq. (5), individual update of $\boldsymbol{x}_{\boldsymbol{i}}$ and $\boldsymbol{v}_{\boldsymbol{i}}$ is implemented about. If $\boldsymbol{x}_{\boldsymbol{i}}$ is selected $T C_{i}$ will plus 1 , if $\boldsymbol{v}_{\boldsymbol{i}}$ is selected $T C_{i}$ will be initialized.

$$
\begin{gathered}
v_{i k}^{g}=x_{i k}^{g}+\phi_{i k}^{g}\left(x_{i k}^{g}-x_{m k}^{g}\right) \\
\boldsymbol{x}_{\boldsymbol{i}}^{\boldsymbol{g}}= \begin{cases}\boldsymbol{v}_{\boldsymbol{i}}^{g} & \text { if } f_{v_{i}^{g}}>f_{x_{i}^{g}} \text { then } T C_{i}=0 \\
\boldsymbol{x}_{\boldsymbol{i}}^{g} & \text { others then } T C_{i}=T C_{i}+1\end{cases}
\end{gathered}
$$

In Eq. (4) and (5), $i$ is the number of individuals, $k$ is the dimension number decided randomly. $g$ is the number of generations, $m$ is the individual number without $i . \phi$ is the random number within the range of $[-1 \sim 1], f_{i}$ shows the fitness of $i$. According to the results obtained by the employed bees, the relative probability $P_{i}^{g}$ can be estimated by Eq. (6). Using the probability, an individual $i^{g}$ can be selected by roulette wheel selection. The location updating will be looped for $\mathrm{N}$ times.

$$
P_{i}^{g}=f_{i}^{g} / \sum_{n=1}^{N} f_{n}^{g}
$$

When the update cannot be implemented to new solutions, the corresponding bee becomes the scout bees. The searching equation is shown as the follows.

$$
x_{i j}=x_{\min }+r_{i j}^{g}\left(x_{\max }-x_{\min }\right) \text { if } T C_{i} \geq \text { limit }
$$

$x_{\text {max }}$ is the maximum value in the search space, $x_{\text {min }}$ is the minimum one. $r$ is a random number between $[0,1]$.

In one iteration, when the best solution $\boldsymbol{x}_{\text {best }}^{\boldsymbol{g}}$ with higher fitness than the one of best solution in previous step, the best solution is updated as the following equation shows. 


$$
\boldsymbol{x}_{\text {best }}= \begin{cases}x_{\text {best }}^{g} & \text { if } f_{x_{\text {best }}^{g}}>f_{x_{\text {best }}} \\ \boldsymbol{x}_{\text {best }} \text { others }\end{cases}
$$

\section{Experiments and Study}

In this research a plant of USM servo system was employed. In the simulation, a step wave signal with the amplitude of 45 degree is introduced as a reference input. The simulation lasts 2000 iteration. Figure 5 shows the response of the proposed method. There was oscillation in the positioning around the steady state. At the end, the positioning converged to 45 degree with smaller error at the steady state. The variation of the gains is shown in Fig. 6. It can be seen that the gains converged smoothly and quickly at around the 400th iteration. Figure 7 shows the convergence in $\mathrm{NN}$ of the proposed method. The weights converged to certain values around 700 iterations. The convergence of the parameters shows the effectiveness of the proposed method in position control.

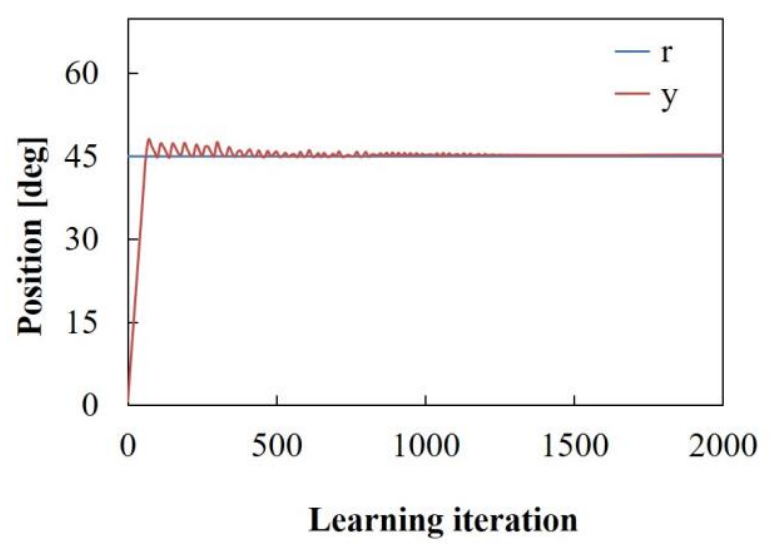

Fig. 5. Response of the proposed control method in position control.

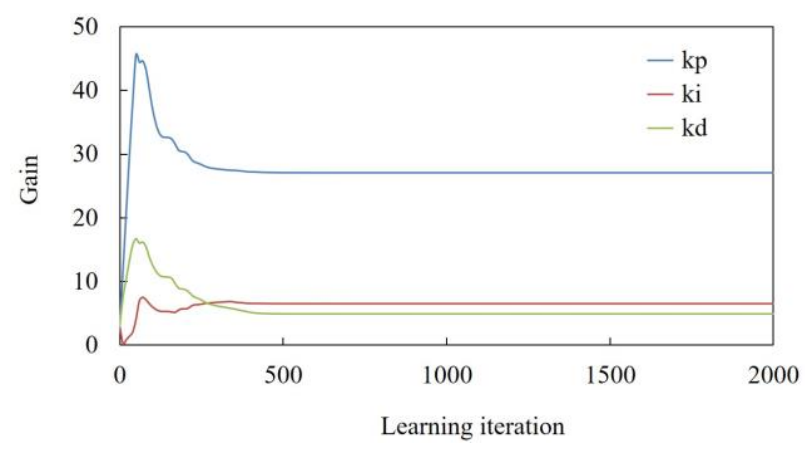

Fig. 6. Variation of PID gains in the proposed method.

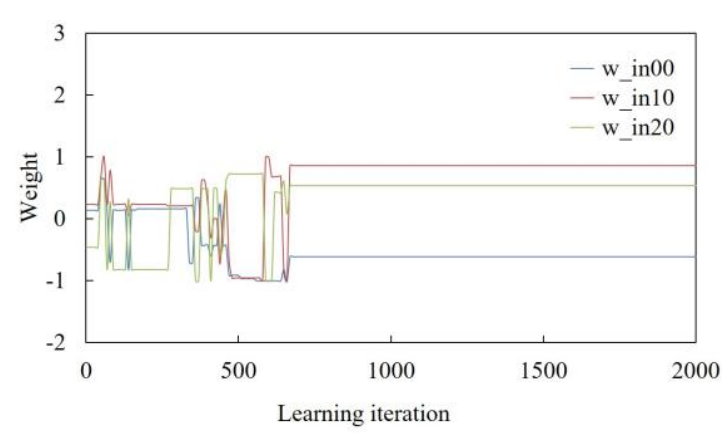

Fig. 7. Variation of the weights in $\mathrm{NN}$ of the proposed method.

\section{Conclusions}

In this paper, a study of intelligent PID control scheme combined with $\mathrm{ABC}$ type $\mathrm{NN}$ has been implemented for USM control. In the proposed method, $\mathrm{NN}$ is employed to update the gains in PID controller. ABC algorithm is applied in NN's learning. The proposed method is able to be applied in compensating characteristic changes of USM. The effectiveness of the proposed method is confirmed by simulation.

\section{References}

(1) K. Astrom, T. Hagglund, C. Hang, and W. Ho: "Automatic tuning and adaptation for PID controllers a survey", Control Engineering Practice, Vol. 1: pp. 699-714, 1993

(2) K. Tanaka, Y. Yoshimura, Y. Wakasa, T. Akashi, M. Oka, and S. Mu: "Variable gain type intelligent PID control for ultrasonic motor" (in Japanese), The Japan Society Applied Electromagnetics and Mechanics, Vol. 17: pp. 107-113, 2009T. Kenjo and T. Sashida: "An Introduction of Ultrasonic Motor" Oxford Science Publications, 1993.

(3) S. Mu and K. Tanaka: "Intelligent IMC-PID control using PSO for ultrasonic motor", International Journal of Engineering Innovation and Management, Vol. 1: pp. 69-76, 2011

(4) K. Uchino: "Piezoelectric ultrasonic motors: Overview", Smart Material Structure, Vol. 7: pp. 273285,1998

(5) C. Zhao: "Ultrasonic Motor - Technologies and Applications", Science Press Beijing and Springer- 
Verlag Berlin Heidelberg, Beijing, 2011

(6) T. Senjyu, H. Miyazato, and K. Uezato: "Position control of ultrasonic motor using neural network" (in Japanese), Transaction of the Institute of Electrical Engineers of Japan D, Vol.116: pp. 1059-1066, 1996

(7) F. Lin, R. Wai, and C. Hong: "Identification and control of rotary traveling-wave type ultrasonic motor using neural networks", IEEE Transactions on Control Systems Technology, Vol. 9: pp. 672-680, 2001

(8) D. Karaboga , B. Basturk: "A powerful and efficient algorithm for numerical function optimization: artificial bee colony (ABC) algorithm", J Glob Optim 39:459-471, 2007 